\title{
ALGEBRA READINESS AND ALGEBRAIC STRUCTURE AS FOUNDATIONAL IDEAS FOR ALGEBRAIC LEARNING
}

\author{
David Feikes, Professor, Purdue University Northwest, feikesd@ pnw.edu \\ William S. Walker III, Assistant Director of CATALYST, Purdue University, wswalker@ purdue.edu \\ Natalie McGathey, Associate Professor, Prairie State College, nmcgathey@ prairiestate.edu \\ Bir Kafle, Associate Professor, Purdue Northwest University, bkafle@pnw.edu
}

\begin{abstract}
This theoretical research brief uses Kaput's (2008) definition of algebra to describe algebra readiness and algebraic structure as they relate to thinking about algebra and thinking with algebra. Algebra readiness and algebraic structure have been core ideas in our curriculum development work to help students develop conceptual understandings of algebra.
\end{abstract}

\section{Algebra, Algebra Readiness, and Algebraic Structure}

Kaput (2008) defines algebra as two core aspects. The first core aspect defines algebra "as systematically symbolizing generalizations of regularities and constraints" (p. 11). This core aspect aligns with our perspective of algebra readiness. Rather than algebra early (Carraher, Schliemann, \& Schwartz, 2008), algebra readiness is developing understandings of fundamental concepts of algebraic thinking like recognizing and analyzing patterns, representing relationships symbolically, and making generalizations. Understanding these concepts allows students to solve algebraic problems using their understandings of arithmetic to interpret and operate on algebraic equations and symbols. Our earlier algebra readiness project, Conceptual Algebra Readiness for Everyone, developed algebra readiness with children in grades 3-7. We characterized this approach as thinking about algebra. Similar to Kaput's first core aspect, the students learned how to generalize and to represent their thinking algebraically.

However, algebra entails more than just generalization and symbolic representation. Kaput (2008) defines the second core aspect of algebra "as syntactically guided reasoning and actions on generalizations expressed in conventional symbols systems" (p.11). Algebra is a manipulable language which allows one to express and operate on generalizations. This core aspect aligns with our perspective of algebraic structure. Algebraic structure is what allows the manipulation of algebraic objects and symbolic generalizations while maintaining algebra's logical consistency. Algebraic structure has provided a foundation for our current Thinking With Algebra (TWA) project and has been a core tenet in the design of curricular materials and the suggested pedagogical approach. For example, the teacher notes include explicit connections to algebraic structure. TWA makes a distinction between thinking about algebra and thinking with algebra. We characterize the latter as manipulating the symbolic generalizations in meaningful ways (Feikes, Kafle, McGathey, \& Walker, 2021). These core aspects of algebra provide connections to learning algebra through algebra readiness and algebraic structure.

\section{References}

Carraher, D., Schliemann, A., \& Schwartz, J. (2008). Early algebra is not the same as algebra early. In J. Kaput, D. W. Carraher, \& M. L. Blanton (Eds.), Algebra in the Early Grades (pp. 235-272). New York: Lawrence Erlbaum Associates.

Feikes, D., Kafle, B., McGathey, N., \& Walker, W. S., III. (2021). Thinking with algebra: A project and perspective. In W. S. Walker, III, L. A. Bryan, S. S. Guzey, \& E. Suazo-Flores (Eds.), Proceedings of the sixth annual Indiana STEM Education Conference. West Lafayette, IN.

Kaput, J. (2008). What is algebra? What is algebraic reasoning? In J. Kaput, D. W. Carraher, \& M. L. Blanton (Eds.), Algebra in the Early Grades (pp. 5-18). New York: Lawrence Erlbaum Associates. 